\section{Cureus}

Received 05/23/2019

Review began 05/25/2019

Review ended 05/28/2019

Published 06/05/2019

\section{(c) Copyright 2019}

Bath et al. This is an open access article distributed under the terms of the Creative Commons Attribution License CC-BY 3.0., which permits unrestricted use, distribution, and reproduction in any medium, provided the original author and source are credited.

\title{
Slit in the Coronaries: A Case of Spontaneous Coronary Artery Dissection
}

\author{
Anandbir S. Bath ${ }^{1}$, Sourabh Aggarwal ${ }^{2}$, Vishal Gupta ${ }^{3}$, Jagadeesh K. Kalavakunta ${ }^{3}$ \\ 1. Internal Medicine, Western Michigan University Homer Stryker M.D. School of Medicine, Kalamazoo, \\ USA 2. Interventional Cardiology, Ascension Borgess Hospital, Kalamazoo, USA 3. Cardiology, Ascension \\ Borgess Hospital, Kalamazoo, USA
}

$\square$ Corresponding author: Anandbir S. Bath, bathanand@gmail.com

Disclosures can be found in Additional Information at the end of the article

\section{Abstract}

Spontaneous coronary artery dissection (SCAD) is a noniatrogenic epicardial coronary artery dissection unrelated to an atherosclerotic disease process. SCAD is responsible for a small percentage of acute coronary syndrome (ACS) cases. The left anterior descending (LAD) artery is the most common artery affected in SCAD, although any coronary artery can be affected. We present an interesting case of SCAD presenting as an ST-elevation myocardial infarction complicated with dissection extending to the left main and distal LAD requiring emergent coronary artery bypass grafting. Our case emphasizes the importance of considering SCAD as a cause for ACS, especially in young patients with minimal atherosclerotic risk factors. Also, a very high recurrence rate demands strict follow-up and multidisciplinary decision making in the population impacted with this rare entity.

Categories: Cardiology, Internal Medicine

Keywords: spontaneous coronary artery dissection (scad), acute coronary syndrome (acs), interventional cardiology

\section{Introduction}

Spontaneous coronary artery dissection (SCAD) is defined as a noniatrogenic epicardial coronary artery dissection that is not related to atherosclerotic plaque rupture/erosion [1]. SCAD is a rare and uncommon cause of acute coronary syndrome (ACS), which can have varied presentations such as myocardial infarction, ventricular arrhythmias, and sudden cardiac death with $2 \%-5 \%$ of the cases presenting as cardiogenic shock [2-6]. We present an interesting case of SCAD presenting as an ST-elevation myocardial infarction (STEMI) complicated with dissection extending to the left main and distal left anterior descending (LAD) artery requiring emergent coronary artery bypass grafting (CABG).

\section{Case Presentation}

A 48-year-old man with a known history of hypertension and no known cardiac history presented to the ED with substernal chest pain radiating to the left jaw, axilla, and arm. It was sudden in onset; he reported the pain started while he was mowing his grass and was associated with shortness of breath and diaphoresis. He had no personal history of diabetes mellitus, hyperlipidemia, smoking, or use of illicit drugs and denied any family history of premature coronary artery disease. His vital signs were significant for elevated blood pressure of 202/126 mmHg with oxygen saturation $>95 \%$ on room air. He was anxious and otherwise had an unremarkable examination with normal S1 and S2 with no murmurs, rubs, or gallops and no rales or rhonchi. 


\section{Cureus}

An electrocardiogram revealed ST elevations in lead I, V4-V6 suggesting anterolateral STEMI. He was loaded with aspirin $325 \mathrm{mg}$ oral and 5000 units of IV heparin. He was emergently transferred to the cardiac catheterization laboratory for angiography. His coronary angiogram was done via the right femoral route and revealed right dominant coronary anatomy with no angiographic evidence of obstructive disease in the right coronary artery and left circumflex artery. His mid LAD had filling defect suspicious for plaque rupture and thrombosis (Figure 1).

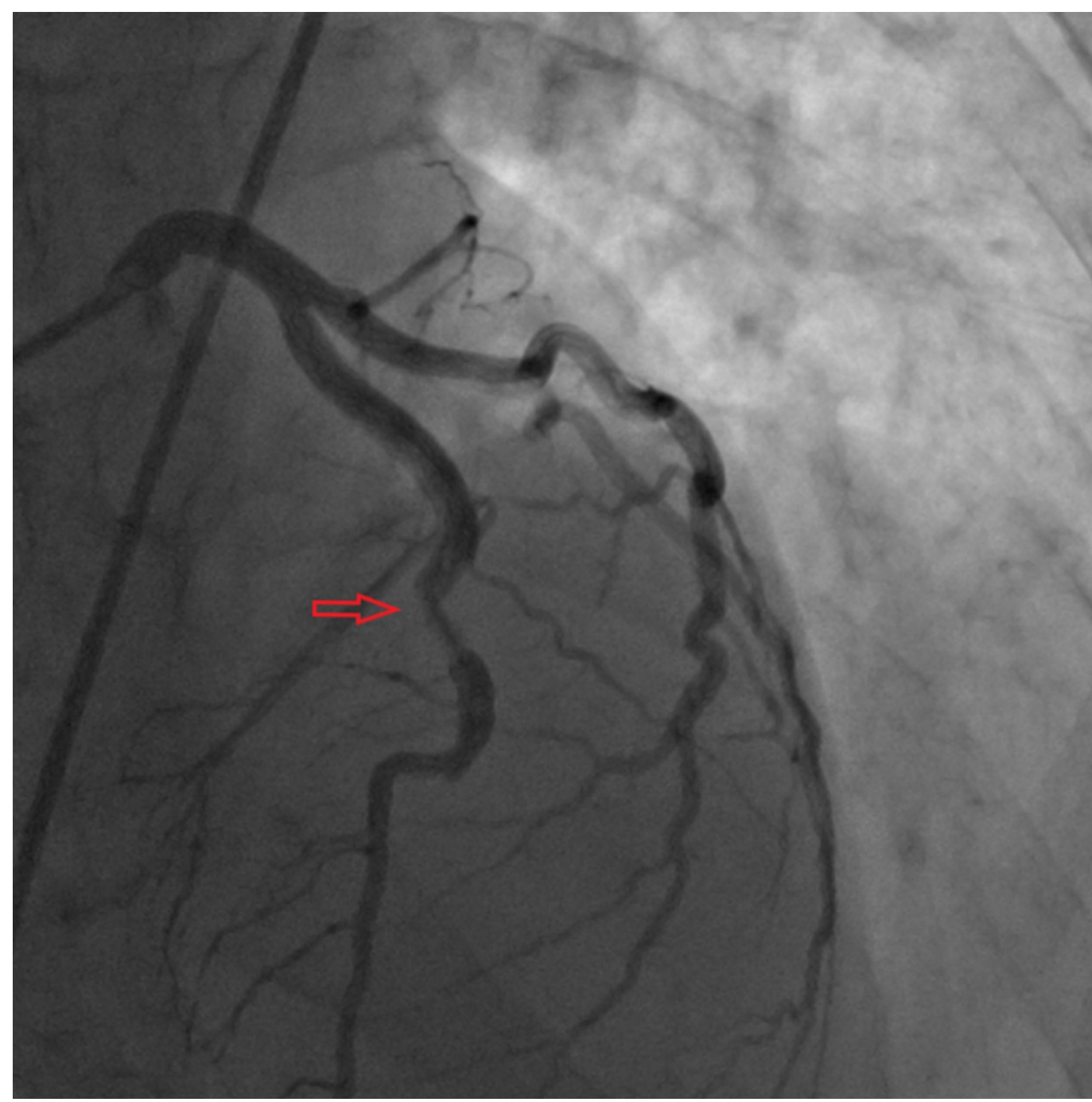

\section{FIGURE 1: Filling defect noted in middle left anterior descending artery.}

After administering additional heparin to achieve activated clotting time $>250$, left main was engaged with extra backup (XB) 3.5 guide catheter and short runthrough wire advanced to the distal LAD. The wire advanced easily, and we navigated to the septal and diagonal branches, confirming the intraluminal location of the wire. On follow-up angiogram, we noted dissection in his middle LAD extending to the diagonal branch, confirming the diagnosis of SCAD.

We initially tried to wire the diagonal branch; however, we were unable to advance the wire to the diagonal branch. We then deployed a drug-eluting stent to seal the proximal end of dissection with a $3.5-\mathrm{mm} \times 18-\mathrm{mm}$ zotarolimus-eluting stent, and we used a $2.5-\mathrm{mm} \times 23-\mathrm{mm}$ zotarolimus-eluting stent to seal the distal end of the dissection (Figure 2). 


\section{Cureus}

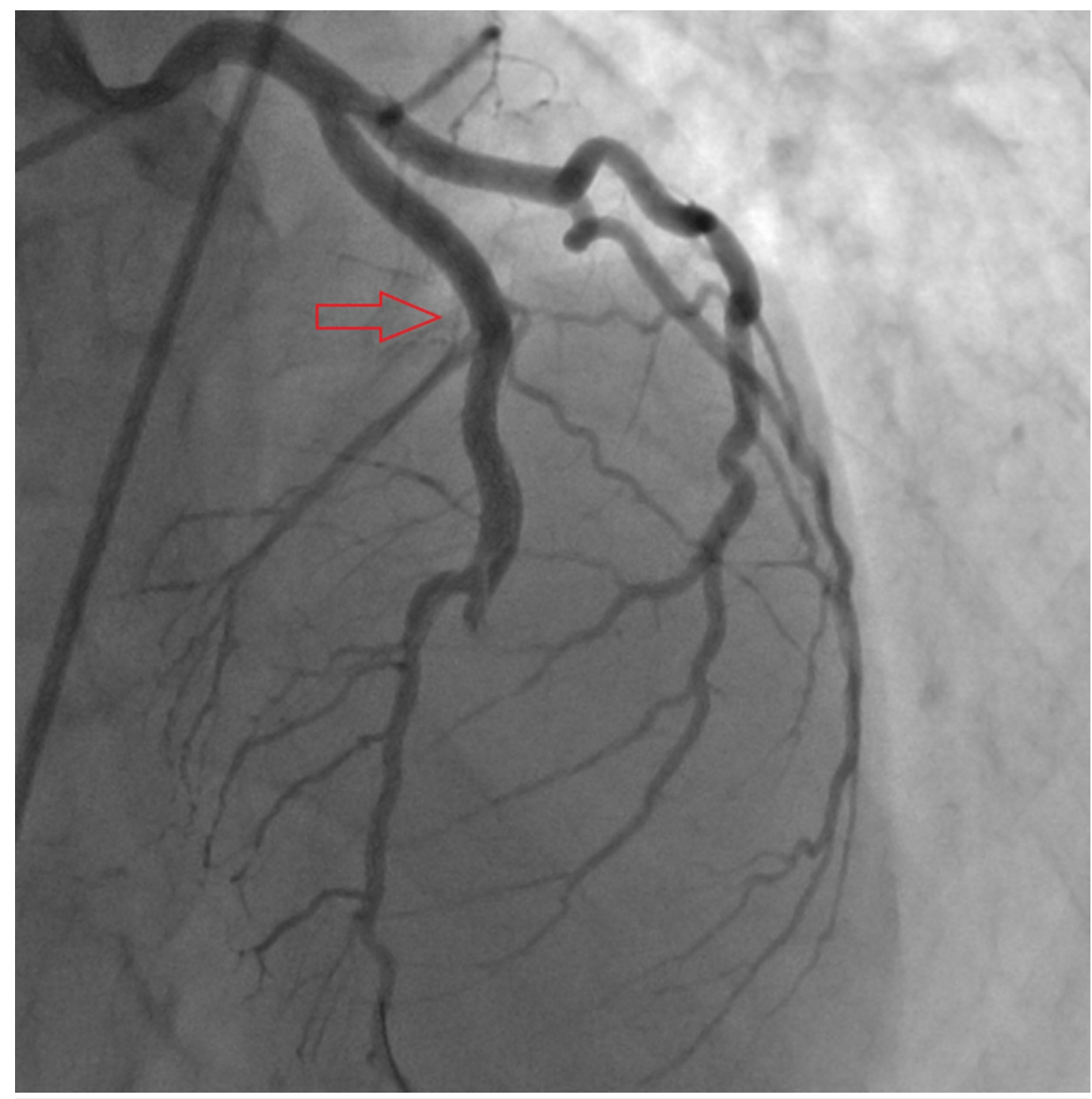

FIGURE 2: Proximal end of dissection sealed with drug-eluting stent.

On follow-up angiogram, we noted the proximal dissection extended to the ostial LAD involving the distal left main artery (Figure 3). 


\section{Cureus}

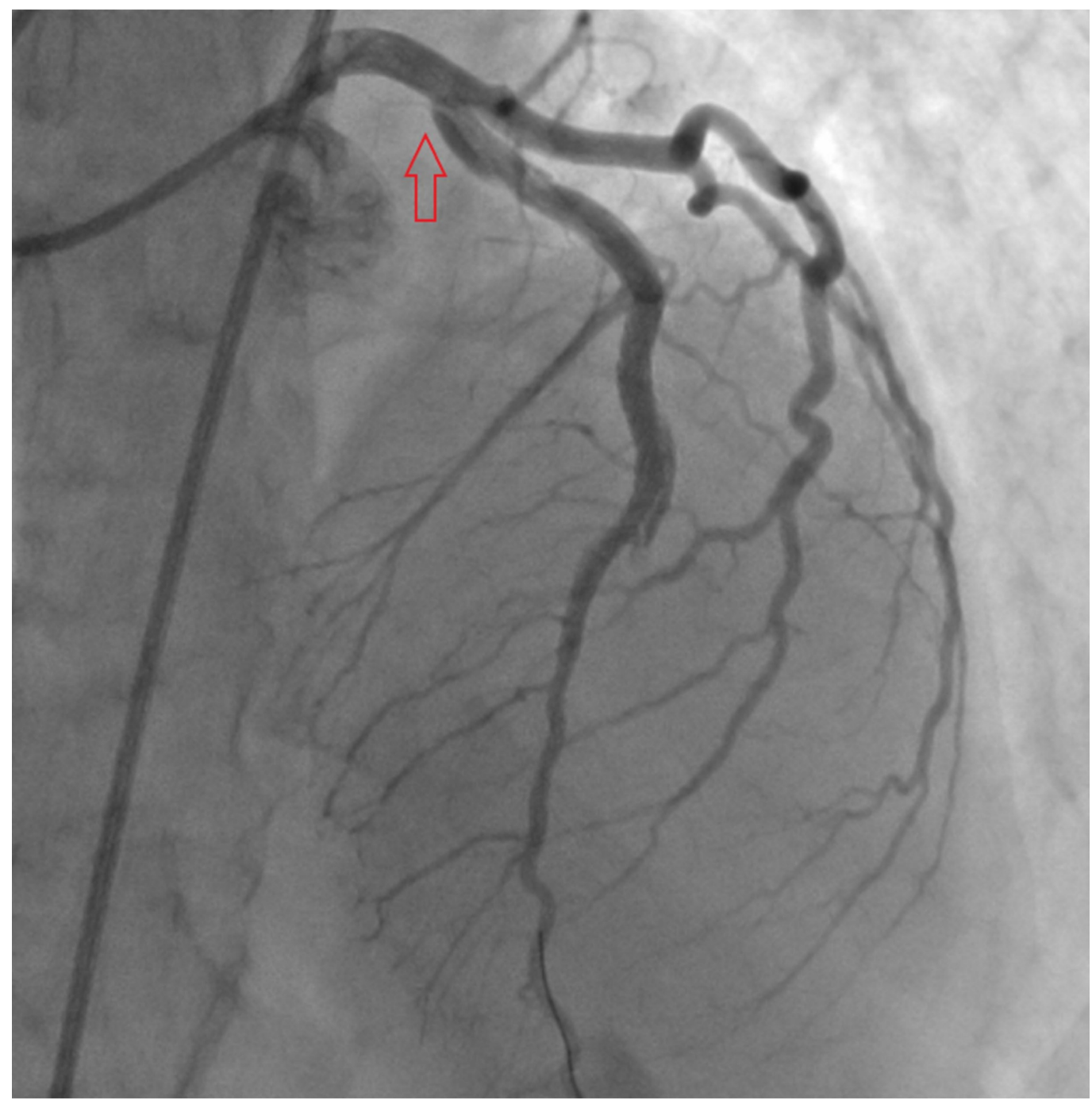

FIGURE 3: Proximal dissection extending into ostial left anterior descending artery involving the left main coronary artery.

At this point, we were concerned that further intervention might lead to a further extension of the dissection proximally, jeopardizing flow to the left main/left circumflex. We consulted with the CT surgical team and discussed the case with them in detail. The patient was emergently taken to the OR for CABG and successfully underwent three-vessel CABG with left internal mammary artery to LAD, aorto-coronary saphenous vein graft to diagonal branch and aortocoronary saphenous vein graft to the obtuse marginal branch. The patient had an unremarkable postoperative course, was extubated the day after the procedure, and went home after three days. He was seen in the clinic for follow-up evaluation and was doing fine.

\section{Discussion}

We present an interesting case of SCAD that presented with STEMI complicated by extension of the dissection proximally, necessitating emergent CABG. SCAD was initially diagnosed in 1931 during the autopsy of a 42-year-old woman. Originally thought to be prevalent in women, SCAD is usually seen in individuals with few traditional atherosclerotic risk factors. While women comprise most cases, men usually present at a younger age than their counterparts with a mean age of 49 [7-8]. Its pathogenesis involves the formation of an intramural hematoma 
(IMH) within the arterial wall, which further compresses the arterial lumen and leads to decreased blood flow. Two mechanisms have been described for the formation of IMH depending on the place of arterial wall separation. Both intimal tear and vasa vasorum rupture lead to pooling of blood in the intramural space, creating a false lumen. Atherosclerotic dissection of the coronaries can differ from the SCAD based on its limited nature of propagation [2]. In our case, the initial appearance was concerning for atherosclerotic plaque rupture; however, the easy extension of the dissection proximally and distally was more consistent with SCAD.

Common conditions which predispose to SCAD include fibromuscular dysplasia, pregnancy, multiparity, inherited arteriopathies, connective tissue disorders, exogenous hormone use, systemic inflammatory disease, and coronary artery spasm. More than $50 \%$ of these patients recall a precipitating factor such as intense exercise, intense Valsalva maneuver, retching, vomiting, intense emotional stress, labor, and recreational drug abuse. Chest pain is the presenting symptom in the majority of the cases with most presenting as ACS [2, 9-11]. Studies that exclude iatrogenic, traumatic, and atherosclerotic dissection suggest that SCAD may be a cause of up to $1 \%-4 \%$ of ACS cases overall, and SCAD is the most common cause of pregnancyassociated myocardial infarction (MI; 43\%) and up to 35\% of MIs in women aged 50 years or younger. The LAD is the most common artery affected in SCAD, although any coronary artery can be affected. In a recent case series, $26 \%$ of cases were found to present as STEMI and 3.6\% with ventricular arrhythmia [12].

Missed diagnosis on angiography and lack of clinical familiarity account for the underdiagnoses of SCAD cases. Intracoronary imaging techniques like intravascular ultrasound (IVUS) and optical coherence tomography (OCT) have increased the detection rates for SCAD, but these are not needed to make the diagnosis [13-15]. However, operators must be careful as intracoronary placement of IVUS catheter and contrast injection during OCT imaging can cause an extension of the dissection. Because the clinical course of our case was consistent with SCAD, we elected not to intervene/interrogate further with intravascular imaging. Three distinct angiographic patterns have been described to help with diagnosis [16]. The appearance of multiple lumens (true and false) on contrast dye staining constitutes type I SCAD. Type II SCAD is characterized by diffuse stenosis of varying severity. Type III SCAD differs from the other two due to focal stenosis and its similarities with atherosclerosis, often requiring intracoronary imaging for its diagnosis. However, a high index of suspicion is needed for diagnosis.

The most recent position statement from the American Heart Association recommended conservation therapy for clinically stable patients with no high-risk features on angiography. However, percutaneous intervention or CABG is recommended in patients with active/ongoing ischemia or hemodynamic instability and CABG in stable patients with involvement of the left main and/or proximal two vessel dissection [1]. Spontaneous healing occurs in most of the cases, suggesting time-dependent healing, but extensive inpatient monitoring is recommended. Interestingly, guidelines recommend the use of the femoral route for angiography as there is a higher rate of iatrogenic dissection in patients with SCAD, and more with the radial route. Hemodynamic stability can dictate further management with unstable patients requiring urgent percutaneous intervention or CABG. In a recent review article, $84 \%$ of patients were managed conservatively, $15 \%$ with percutaneous intervention, and $1 \%$ required urgent CABG [17]. Complications such as the iatrogenic extension of the dissection and true lumen occlusion should be considered before proceeding with the early invasive strategy [56,18-19]. Dual antiplatelet therapy for one year is recommended after the successful percutaneous intervention [20].

Our patient presented with an ST-elevation in the anterolateral leads and had ongoing chest pain, which prompted an urgent revascularization course. He had type I SCAD on angiography. We attempted to stabilize the dissection once the guidewire was confirmed in the true lumen. 
The placement of the stent led to proximal and distal extension of the dissection requiring multiple stent placements and urgent cardiothoracic consultation for emergent CABG.

\section{Conclusions}

The diagnosis of SCAD should be considered in all cases of ACS presenting in young patients with minimal traditional atherosclerotic risk factors. Hemodynamic and clinical stability should dictate the management strategies unless the patient presents with ischemic changes where urgent intervention is needed for revascularization. The 50\% recurrence rate demands strict follow-up evaluation, and multidisciplinary decision making is necessary for favorable outcomes in patients impacted with this rare entity.

\section{Additional Information Disclosures}

Human subjects: Consent was obtained by all participants in this study. Conflicts of interest: In compliance with the ICMJE uniform disclosure form, all authors declare the following: Payment/services info: All authors have declared that no financial support was received from any organization for the submitted work. Financial relationships: All authors have declared that they have no financial relationships at present or within the previous three years with any organizations that might have an interest in the submitted work. Other relationships: All authors have declared that there are no other relationships or activities that could appear to have influenced the submitted work.

\section{References}

1. Hayes SN, Kim ESH, Saw J, et al.: Spontaneous coronary artery dissection: current state of the science: a scientific statement from the American Heart Association. Circulation. 2018, 137:523-557. 10.1161/CIR.0000000000000564

2. Saw J: Spontaneous coronary artery dissection. Can J Cardiol. 2013, 29:1027-1033. 10.1016/j.cjca.2012.12.018

3. Bergen E, Huffer L, Peele M: Survival after spontaneous coronary artery dissection presenting with ventricular fibrillation arrest. J Invasive Cardiol. 2005, 17:4-6.

4. Mortensen KH, Thuesen L, Kristensen IB, Christiansen EH: Spontaneous coronary artery dissection: a Western Denmark Heart Registry study. Catheter Cardiovasc Interv. 2009, 74:710-717. 10.1002/ccd.22115

5. Nakashima T, Noguchi T, Haruta S, et al.: Prognostic impact of spontaneous coronary artery dissection in young female patients with acute myocardial infarction: areport from the Angina Pectoris-Myocardial Infarction Multicenter Investigators in Japan. Int J Cardiol. 2016, 207:341-348. 10.1016/j.ijcard.2016.01.188

6. Lettieri C, Zavalloni D, Rossini R, et al.: Management and long-term prognosis of spontaneous coronary artery dissection. Am J Cardiol. 2015, 116:66-73. 10.1016/j.amjcard.2015.03.039

7. Eckart RE, Shry EA, Burke AP, et al.: Sudden death in young adults: an autopsy-based series of a population undergoing active surveillance. J Am Coll Cardiol. 2011, 58:1254-1261. 10.1016/j.jacc.2011.01.049

8. Fahmy P, Prakash R, Starovoytov A, Boone R, Saw J: Pre-disposing and precipitating factors in men with spontaneous coronary artery dissection. JACC Cardiovasc Interv. 2016, 9:866-868. 10.1016/j.jcin.2016.02.024

9. Tokura M, Taguchi I, Kageyama M, et al.: Clinical features of spontaneous coronary artery dissection. J Cardiol. 2014, 63:119-122. 10.1016/j.jjcc.2013.07.001

10. Saw J, Poulter R, Fung A, Wood D, Hamburger J, Buller CE: Spontaneous coronary artery dissection in patients with fibromuscular dysplasia: a case series. Circ Cardiovasc Interv. 2012, 5:134-137. 10.1161/CIRCINTERVENTIONS.111.966630

11. DeMaio SJ, Kinsella SH, Silverman ME: Clinical course and long-term prognosis of spontaneous coronary artery dissection. Am J Cardiol. 1989, 64:471-474. 10.1016/00029149(89)90423-2 
12. Saw J, Aymong E, Sedlak T, et al.: Spontaneous coronary artery dissection: association with predisposing arteriopathies and precipitating stressors and cardiovascular outcomes. Circ Cardiovasc Interv. 2014, 7:645-655. 10.1161/CIRCINTERVENTIONS.114.001760

13. Maehara A, Mintz GS, Castagna MT, et al.: Intravascular ultrasound assessment of spontaneous coronary artery dissection. Am J Cardiol. 2002, 89:466-468. 10.1016/S00029149(01)02272-X

14. Alfonso F, Paulo M, Gonzalo N, et al.: Diagnosis of spontaneous coronary artery dissection by optical coherence tomography. J Am Coll Cardiol. 2012, 59:1073-1079. 10.1016/j.jacc.2011.08.082

15. Kalavakunta JK, Hajjar M, Chandrashekar R, Agrawal Y, Gourineni N, Fischell TA: Role of imaging in spontaneous coronary artery dissection - a report and review of literature. Eur J Arrhythmia Electrophysiol. 2016, 2:62-65. 10.17925/EJAE.2016.02.02.62

16. Saw J: Coronary angiogram classification of spontaneous coronary artery dissection. Catheter Cardiovasc Interv. 2014, 84:1115-1122. 10.1002/ccd.25293

17. Saw J, Starovoytov A, Humphries K, et al.: Canadian spontaneous coronary artery dissection cohort study: in-hospital and 30-day outcomes. Eur Heart J. 2019, 40:1188-1197. 10.1093/eurheartj/ehz007

18. Tweet MS, Hayes SN, Pitta SR, et al.: Clinical features, management, and prognosis of spontaneous coronary artery dissection. Circulation. 2012, 126:579-588. 10.1161/CIRCULATIONAHA.112.105718

19. Alfonso F, Paulo M, Lennie V, et al.: Spontaneous coronary artery dissection: long-term follow-up of a large series of patients prospectively managed with a "conservative" therapeutic strategy. JACC Cardiovasc Interv. 2012, 5:1062-1070. 10.1016/j.jcin.2012.06.014

20. Lempereur M, Fung A, Saw J: Stent mal-apposition with resorption of intramural hematoma with spontaneous coronary artery dissection. Cardiovasc Diagn Ther. 2015, 5:323-329. 10.3978/j.issn.2223-3652.2015.04.05 